\title{
Age-related Differeneces in the Effect of Acute Hyperglycemia on Myocardial Ischemia-Reperfusion Injury
}

\author{
Jong-Kwang Park, Sung Yeon Ham and Yon Hee Shim \\ Yonsei University College of Medicine, Seoul, Republic of Korea
}

\section{Background}

Age and acute hyperglycemia are known risk factors for myocardial ischemia-reperfusion injury and combined frequently in clinical circumstances. However, there are limited data to examine myocardial ischemia-reperfusion injury in animal models exhibiting these co-morbidities concomitantly. Thus, we investigated the effect of acute hyperglycemia on myocardial ischemia-reperfusion injury in different age-groups and possible mechanisms.

\section{Methods}

Three different age groups of male Sprague Dawley rats were included (young-aged, 3 months; middle-aged, 10-12 months; and old-aged, 20-22 months). Rats received $1.2 \mathrm{~g} / \mathrm{kg}$ of dextrose (hyperglycemic group) or same volume of normal saline (normoglycemic group) according to the group. Rats were subjected to coronary artery occlusion for $45 \mathrm{~min}$ followed by reperfusion for $240 \mathrm{~min}$ (fig.1). By measuring the infarct size and ejection fraction, we estimated the susceptibility to ischemiareperfusion injury in rat. Proteins related to apoptosis (C-PARP, Bcl-2, Bax, and cytochrome C) and autophagy (Bnip3, Beclin-1, Atg5, and LC3B-II) were evaluated by western blot assay.

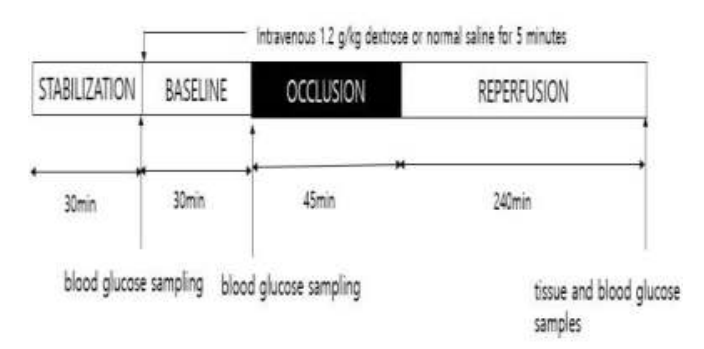

Fig 1. Experimental protocol. Rats were divided into 3 groups according to the age; young $(\mathrm{Y}, 3 \mathrm{mo})$, middle (M, 10-12mo) and old (O, 20-24mo). Each age group of rats were randomly assigned to 4 groups; normoglycemia + sham (NG), hyperglycemia +sham (HG), normoglycemia + ischemia-reperfusion injury (NG+IR) and hyperglycemia $+\mathrm{IR}$ $(H G+I R)$.

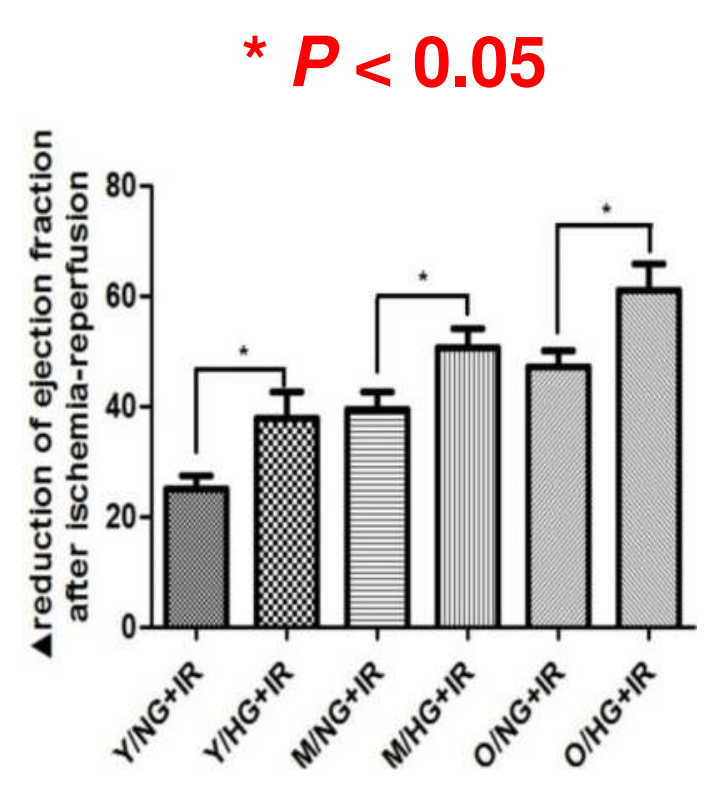

Fig 2. Changes of LV ejection fraction after ischemia-reperfusion.

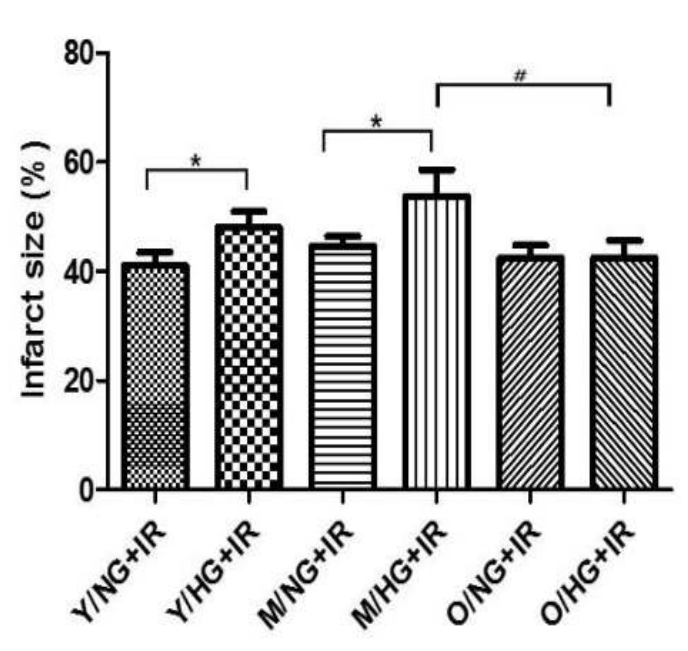

Fig 3. Myocardial infarct size expressed as a percentage of LV area at risk.

\section{Results}

While reduction of ejection fraction after ischemia-reperfusion was aggravated by acute hyperglycemia in all age groups (fig.2), infarct size was increased by acute hyperglycemia in young- and middle-aged rats but not in old-aged rats (fig.3). Acute hyperglycemia increased expression of Bnip3 and Beclin-1 after ischemia-reperfusion in young- and middle-aged rats but not in old-aged rats. Also, increased expression of Bax, Cytochrome C, Atg5, and LC3B-II by acute hyperglycemia occurred only in young- or middle-aged rats (fig $4 \& 5$ ).

\section{Conclusion}

The results of current study demonstrated that acute hyperglycemia did not aggravate myocardial ischemiareperfusion injury in old-aged rats, unlike young and middle-aged rats. This heterogeneity may be a consequence of attenuation in changes of apoptotic and autophagic signalling after ischemiareperfusion injury under acute hyperglycemia in old-aged heart.
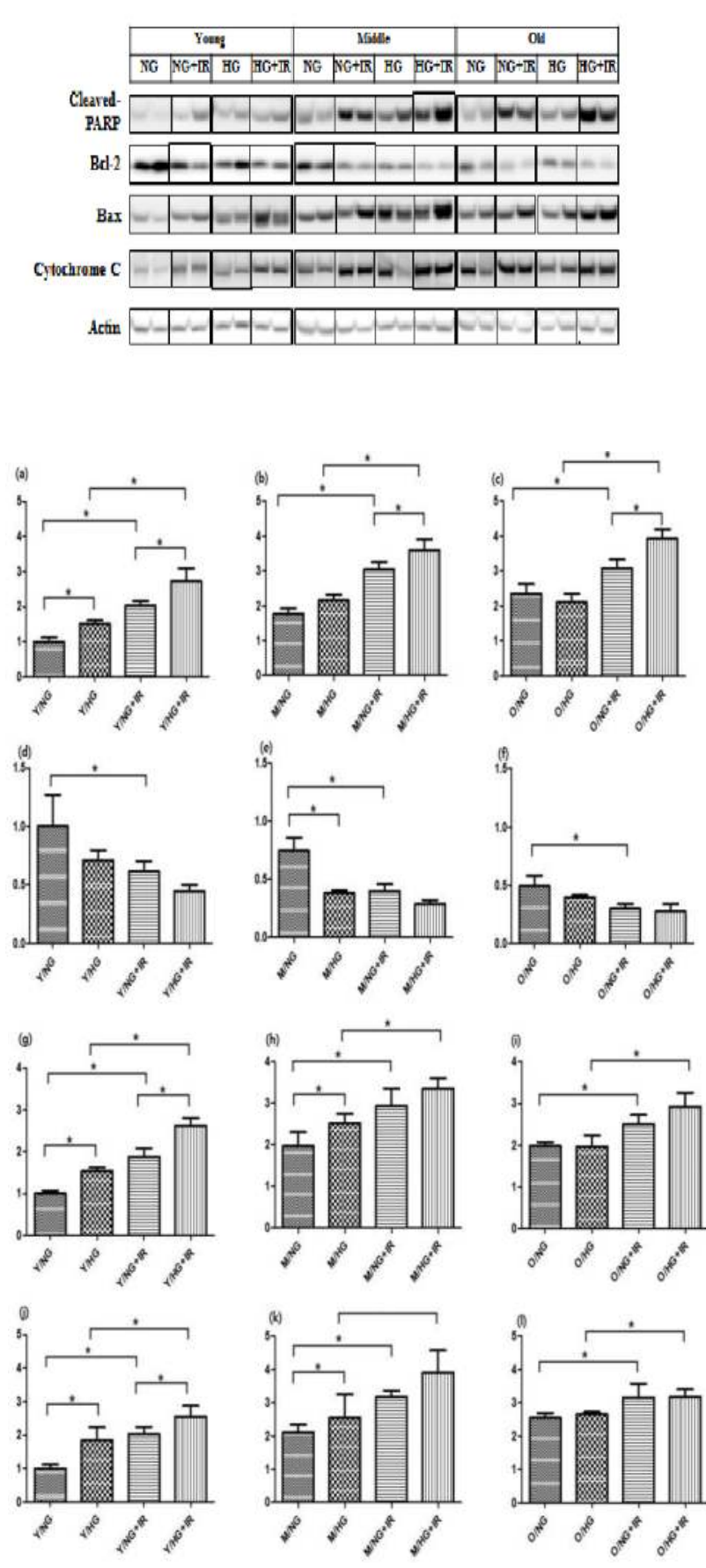

:

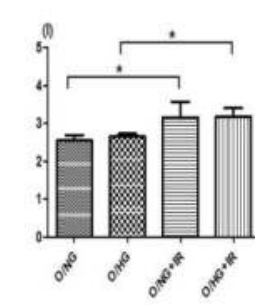

Fig 4. The expression of proteins related to apoptosis. $(a, b, c)$ C-PARP, $(d, e, f) B c l-2$, $(\mathrm{g}, \mathrm{h}, \mathrm{i})$ Bax $(\mathrm{j}, \mathrm{k}, \mathrm{l})$ Cytochrome C.
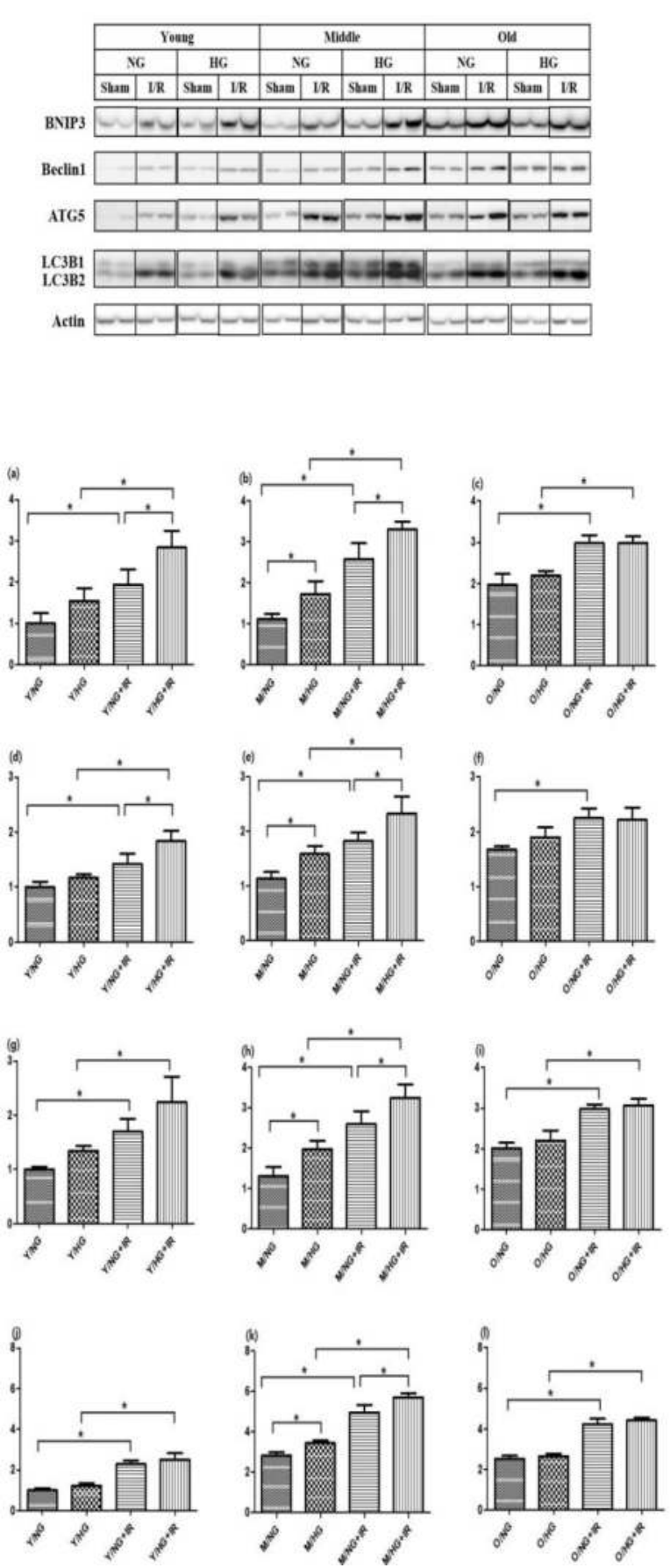

Fig 5. The expression of proteins related to autophagy. $(a, b, c)$ Bnip3, (d,e,f) Beclin-1, (g,h,i) Atg5, (j,k,l) LC3B-II. 